\title{
Very organic-rich bodies in the inner and outer solar system
}

\author{
M.Yu. ZOLOTOV
}

School of Earth and Space Exploration, Arizona State

University, Tempe, AZ 85287-1404, USA

(zolotov@asu.edu)

Although the solar $\mathrm{C} / \mathrm{Si}$ atomic ratio of $\sim 9.5$ suggests abundant carbon-rich bodies, terrestrial planets and meteorites are rich in silicates and metals. Even carbonaceous chondrites with abundant insoluble organic matter (IOM) have $\mathrm{C} / \mathrm{Si}<0.8$. Until recently, only dust of comets $1 \mathrm{P} /$ Halley $(\mathrm{C} / \mathrm{Si}=3.4-5.4)$ and $67 \mathrm{P} / \mathrm{C}-\mathrm{G}(\mathrm{C} / \mathrm{Si}=4.3-6.9)$, and some anhydrous interplanetary dust particles $(\mathrm{C} / \mathrm{Si}=0.7-4.9)$ revealed more abundant organic matter $(\mathrm{OM})$ than chondrites. The detection of $\sim 8-14 \mathrm{wt} \% \mathrm{C}$ in Ceres' surface materials with the Dawn spacecraft suggests $7-16 \mathrm{wt} \%$ and up to 30 vol\% of IOM-like material in an ice-less interior with $\mathrm{C} / \mathrm{Si}=$ 1.9-3.6 [1]. The similarity of near infrared spectra of Ceres with spectra of some large asteroids [2] suggests a half of the main asteroid belt mass consisted of very $\mathrm{C}$-rich materials that are absent from the meteorite collection. Very C-rich asteroids could have formed at larger heliocentric distances than parent bodies of carbonaceous chondrites. According to the Grand Tack model, both C-type chondritic and very Crich bodies could have migrated from the outer solar system at 5-6 Ma after formation of CAIs. The migration implies abundant $\mathrm{OM}$ within icy moons (e.g. Europa, Enceladus, Triton) and trans-neptunian objects (TNOs) (Pluto, etc.). Interior models for Titan and Ganymede suggest $20 \mathrm{wt} \%$ of OM [3]. Abundant OM within bodies with density of 1.5-2.5 $\mathrm{g} \mathrm{cm}^{-3}$ advocates for relatively water-poor compositions, consistent with the detection of OM rather than water ice on Arrokoth [4] and some other TNO's. Abundant OM affected thermal and physical-chemical evolution of larger and tidallyforced bodies. Alteration of low- and high-molecular weight $\mathrm{OM}$ at variable $T-P-f \mathrm{H}_{2}-a \mathrm{H}_{2} \mathrm{O}$ conditions changed $\mathrm{H} / \mathrm{N} / \mathrm{C} / \mathrm{O}$ ratios in IOM-like materials and led to formation of new compounds. Species released from altered $\mathrm{OM}$ (e.g. $\mathrm{CH}_{4}, \mathrm{~N}_{2}$, $\mathrm{NH}_{3} / \mathrm{NH}_{4}{ }^{+}$) affected interior, surface and atmospheric compositions. Organic-rich (oily) and hydrocarbon clathrate layers/lenses could have formed in addition to solid/liquid water layers and influenced thermal conductivity, viscosity, permeability and water-organic-rock interactions.

[1] Zolotov (2020) Icarus 335, 113404. [2] Rivkin et al. (2019) JGR-Planets 124, 1393-1409. [3] Néri et al. (2020) EPSL 530, 115920. [4] Grundy et al. (2020) Science, $10.1126 /$ science.aay 3705 . 